\title{
The Chip Firing Game and Matroid Complexes
}

\author{
Criel Merino \\ Instituto de Matemáticas, UNAM Área de la investigación científica, Circuito Exterior, Ciudad Universitaria \\ Coyoacán 04510, México, D.F. México.
}

received February 4, 2001, revised April 20, 2001, accepted May 4, 2001.

\begin{abstract}
In this paper we construct from a cographic matroid $M$, a pure multicomplex whose degree sequence is the $h$-vector of the the matroid complex of $M$. This result proves a conjecture of Richard Stanley [Sta96] in the particular case of cographic matroids. We also prove that the multicomplexes constructed are $M$-shellable, so proving a conjecture of Manoj Chari [Cha97] again in the case of cographic matroids. The proofs use results on a game for graphs called the chip firing game.
\end{abstract}

Keywords: Chip-firing game, Tutte polynomial, Simplicial complex

\section{Introduction}

The origins of the chip firing game can be dated back at least to 1983 when Joel Spencer [Spe86] introduced a "balancing game" on graphs that were long paths. Later, in [ALS ${ }^{+} 89$, BLS91], a generalization to general graphs of this process and a careful study of its dynamics were presented. In the game, some chips are put on each vertex of a graph $G$; a vertex is said to be ready if it has at least as many chips as its degree, in which case we can fire it and the result is that it distributes one chip to each of its neighbours, this can cause another vertex to be ready and so on. This game was called the chip firing game.

It was Norman Biggs [Big99a] who came up with a process that was related to this game and to what is known in self-critical systems as Abelian sandpiles. In this game we also have a graph $G$, but this time we are given a special vertex $q$. The rules of this new game are as above for every vertex except for $q$, but $q$ has a debit of chips equal to the number of chips on the graph and is ready only when every other vertex is not, then $q$ is fired until some vertex is ready. The last rule ensures an infinite game. In [Big99a], the game is called the dollar game, and dollars are used instead of chips. Here, however, we stick to the term chip firing game for this new game.

\section{Preliminars}

We consider throughout this paper labelled connected graphs which may have loops and multiple edges, so it is useful for a graph $G$ and a vertex $v \in V(G)$ to denote by indeg $(v)$ twice the number of loops at $v$, and 1365-8050 @ 2001 Maison de l'Informatique et des Mathématiques Discrètes (MIMD), Paris, France 
by $\operatorname{exdeg}(v)$ the number of edges that are incident to $v$ but are not loops, so $\operatorname{deg}(v)=\operatorname{indeg}(v)+\operatorname{exdeg}(v)$. Also, for $v, w \in V(G)$, we define $v(v, w)$ to be the number of edges joining $v$ and $w$.

A matroid $M$ is just a generalization of a matrix and can be defined as a pair $(E, r)$, where $E$ is a finite set and $r$ is a submodular rank function mapping $2^{|E|} \rightarrow \mathbb{Z}$ and satisfying the conditions

$$
\begin{array}{clrl}
0 \leq r(A) \leq|A|, & & A \subseteq E, \\
A \subseteq B \Rightarrow r(A) \leq r(B), & & \text { and } \\
r(A \cup B)+r(A \cap B) \leq r(A)+r(B), & & A, B \subseteq E .
\end{array}
$$

If $A \subseteq E$ and $r(A)=|A|$, the set $A$ is called an independent set of $M$, and a maximal independent set in the poset of subsets of $E$ is called a basis of $M$. It is not difficult to prove, see [Ox192], that if $A \subseteq B$ and $B$ is an independent set, then $A$ is also an independent set of $M$; and that any basis of $M$ has cardinality $r(E)$. Also, for a matroid $M=(E, r)$ we have its dual matroid $M^{*}=\left(E, r^{*}\right)$, where the rank function is defined by

$$
r^{*}(A)=|A|-r(E)+r(E \backslash A),
$$

for all $A \subseteq E$. It is easy to prove that $\left(M^{*}\right)^{*}=M$, see [Ox192].

As examples of matroids we have graphic matroids: given a graph $G=(V, E)$ we have the matroid $M(G)=(E, r)$, where for a subset of edges $A$ its rank is defined by

$$
r(A)=|V|-k(A),
$$

and $k(A)$ is the number of connected components in the subgraph of $G$ with edge set $A$ and vertex set $V$. Clearly, if $G$ is connected, the bases of $M(G)$ are the spanning trees of $G$ and $r(E)=|V|-1$. A matroid $M$ whose dual matroid $M^{*}$ can be realised as a graphic matroid is called a cographic matroid. If the cographic matroid $M=(E, r)$ has as dual the graphic matroid $M^{*}=N(G)$, then $r(E)$ is equal to the cyclomatic number of $G$. For more about matroid theory see [Ox192, Wel76].

If $M$ is a matroid over a set $E$, then its Tutte polynomial is defined as

$$
T(M ; x, y)=\sum_{A \subseteq E}(x-1)^{r(E)-r(A)}(y-1)^{|A|-r(A)},
$$

where $r$ is the rank function of $M$. An interesting property of $T$ that relates $M$ and $M^{*}$ is that one has $T\left(M^{*} ; x, y\right)=T(M ; y, x)$. This polynomial is an important invariant as it contains much information on the matroid, for example, $T(M ; 1,1)$ is equal to the number of bases of $M$. See [BO92] for a comprehensive survey.

\section{The chip firing game}

We restate the definition of the chip firing game with some mathematical notation. Let $G$ be a graph and $q \in V(G)$. A configuration is a function

$$
\theta: V(G) \rightarrow \mathbb{Z}
$$

where $\theta(v) \geq 0$ for all $v \neq q$ and $\theta(q)=-\sum_{v \neq q} \theta(v)$. A vertex $v \neq q$ is ready if $\theta(v) \geq \operatorname{deg}(v), q$ is ready if every other vertex is not ready. For a configuration $\theta$ and a ready vertex $w$, firing $w$ results in the new configuration $\theta^{\prime}$ defined by:

$$
\theta^{\prime}(v)= \begin{cases}\theta(v)+v(v, w), & \text { if } v \neq w, \\ \theta(w)-\operatorname{exdeg}(w), & \text { if } v=w .\end{cases}
$$


Notice that if we have a loop at vertex $w$, a firing of the vertex $w$ sends two chips through the loop that return again to the vertex, so loops do not affect the redistribution of chips.

A legal sequence for a configuration $\theta=\theta_{1}$ is a sequence $\sigma$ of vertices $\left(v_{1}, \ldots, v_{k}\right)$, such that $v_{1}$ is ready in $\theta_{1}$ and at each moment $i, v_{i}$ is ready in $\theta_{i}$, and $\theta_{i}$ is obtained from $\theta_{i-1}$ by firing $v_{i-1}, 2 \leq i \leq k$. So a legal sequence is a sequence of vertices that can be fired in a game with starting configuration $\theta$. If we can go from an initial configuration $\theta$ to a configuration $\theta^{\prime}$ by a legal sequence we write $\theta \rightarrow \theta^{\prime}$. A configuration $\theta$ is stable if for every vertex $v \neq q, \theta(v)<\operatorname{deg}(v)$, so, in a stable configuration, the only vertex that is ready is $q$. Suppose we start with a configuration $\theta$ and after a legal sequence we arrive at the same configuration, we say that $\theta$ is a recurrent configuration. A stable configuration that is also recurrent is called a critical configuration.

\subsection{Critical configurations}

We are going to analyse very closely the critical configurations of a given graph as their structure will be used later in our proofs. The results we are presenting here have been proved in different ways. One approach is to use direct counting arguments as in [Big99a]. Another is to apply group theory arguments to the Picard group as in [CR00].

Theorem 3.1. For a graph $G$ and a configuration $\theta$, there exists a unique critical configuration $c$ such that $\theta \rightarrow c$. In particular, if $\theta$ is critical, then $\theta=c$.

For a critical configuration $\theta$, we called the legal firing of minimal length that makes $\theta$ recur a critical sequences. Critical sequences have been studied before [Big99a, BW97] for loopless graphs with multiple edges. We include a minor extension of Lemma 3.6 from [Big99a, pp. 5] that appears in [Mer97] and we required later.

Lemma 3.2. Let $G$ be a graph and $c$ be a critical configuration, then any critical sequence for $c$ consists of firing all the vertices of $G$ exactly once.

For a configuration $\theta$, we define its weight, $w(\theta)$, to be

$$
w(\theta)=\sum_{v \neq q} \theta(v) .
$$

If $\theta$ is critical, we also define its level as

$$
\operatorname{level}(\theta)=w(\theta)-|E(G)|+\operatorname{deg}(q) .
$$

This definition seems less natural but the following theorem of Biggs [Big99a] tell us that it is actually the right quantity to consider if we want to graded the critical configurations.

Theorem 3.3. Let $G$ be a graph and $\theta$ a critical configuration, then

$$
0 \leq \operatorname{level}(\theta) \leq|E(G)|-|V(G)|+1 .
$$

Notice that the last quantity is the cyclomatic number of $G$.

For a graph $G$, we define, for every $i \geq 0, c_{i}$ to be the number of critical configurations with level $i$. By Theorem 3.3, $c_{i}=0$ for all $i>|E(G)|-|V(G)|+1$. We now take the generating function of the critical configurations, that is, the polynomial

$$
P_{q}(G ; y)=\sum_{i=0}^{|E(G)|-|V(G)|+1} c_{i} y^{i} .
$$


All these definitions were proposed by Biggs [Big99b]. Later in [Mer97] it was proved that the above polynomial is an evaluation of the Tutte polynomial.

Theorem 3.4. For a graph $G$ and a vertex $q$, we have that the generating function of the critical configurations is the Tutte polynomial of $G$ along the line $x=1$, that is,

$$
P_{q}(G ; y)=T(G ; 1, y) .
$$

Thus, the polynomial on the left side is independent of the choice if the special vertex $q$.

\section{A conjecture of Richard Stanley}

\subsection{Simplicial complexes}

In this section we use the chip firing game and its relation with the Tutte polynomial given by Theorem 3.4 to prove a long standing conjecture of Richard Stanley [Sta96] in the particular case of cographic matroids.

Let $\Delta$ be a pure $d$-1-dimensional simplicial complex, that is a $d$-1-dimensional simplicial complex whose maximal faces, or facets, have all the same cardinality. Associated to $\Delta$ we have its face vector or $f$-vector $\left(f_{0}, f_{1}, \ldots, f_{d}\right)$, where $f_{i}$ is the number of faces of size $i$ of $\Delta$. The generating function of the $f$-vector, or face enumerator is defined by

$$
f_{\Delta}(x)=\sum_{i=0}^{d} f_{i} x^{d-i} .
$$

For a pure simplicial complex $\Delta$, a shelling is a linear order of the facets $F_{1}, F_{2}, \ldots, F_{t}$ such that, for $1 \leq l \leq t, F_{l}$ meets the complex generated by its predecessors, called $\Delta_{l-1}$, in a non-void union of maximal proper faces. A complex is said to be shellable if it is pure and admits a shelling.

Define, for $1 \leq l \leq t$,

$$
\mathcal{R}\left(F_{l}\right)=\left\{x \in F_{l} \mid F_{l} \backslash x \in \Delta_{l-1}\right\},
$$

where here $\Delta_{0}=\emptyset$. The number of facets such that $\left|F_{l}-\mathcal{R}\left(F_{l}\right)\right|=i$ is denoted by $h_{i}$ and it does not depend on the particular shelling, see [Bjö92]. The vector $\left(h_{0}, h_{1}, \ldots, h_{d}\right)$ is called the $h$-vector of $\Delta$. The generating function of the $h$-vector, or shelling polynomial is given by

$$
h_{\Delta}(x)=\sum_{i=0}^{d} h_{i} x^{d-i} .
$$

Then, it is well known, see for example [Bjö92], that the face enumerator and the shelling polynomial satisfy the relation

$$
h_{\Delta}(x+1)=f_{\Delta}(x) .
$$

If $M=(E, r)$ is a matroid, the family of all independent sets forms a simplicial complex of dimension $r(E)-1$, that we denote by $\Delta(M)$. The facets of $\Delta(M)$ are the bases of the matroid $M$ and therefore, $\Delta(M)$ is pure. Complexes of this kind are called matroid complexes. Matroid complexes are known to be shellable, see [Bjö92], and it is also known [Bjö92] that the shelling polynomial of $\Delta(M)$ is an evaluation of the Tutte polynomial, that is,

$$
T(M ; x, 1)=h_{\Delta(M)}(x)
$$


and, by duality, we also have

$$
T(M ; 1, y)=h_{\Delta\left(M^{*}\right)}(y) .
$$

\subsection{The main result}

An order ideal ( or down-set) of a poset $P$ is a subset $I$ of $P$ such that if $x \in I$ and $y \leq x$, then $y \in I$. If we take as a poset $P$ the set of all monomials over indeterminates $z_{1}, \ldots, z_{n}$ and the order given by divisibility, then an order ideal of $P$ is called a multicomplex over $z_{1}, \ldots, z_{n}$.

If we form the poset $\left(\mathbb{N}^{n}, \leq\right)$, where $a \leq b$ if $a(i) \leq b(i)$ for $1 \leq i \leq n$, then a multicomplex $\mathcal{M}$ can also be seen as an order ideal of $\left(\mathbb{N}^{n}, \leq\right)$. More explicitly, if $\mathcal{M}$ is a multicomplex over $z_{1}, \ldots, z_{n}$, then the image of the function $\mu: \mathcal{M} \rightarrow \mathbb{N}^{n}$ defined by

$$
\mu\left(z_{1}^{i_{1}}, \ldots, z_{n}^{i_{n}}\right)=\left(i_{1}, \ldots, i_{n}\right)
$$

is an order ideal of $\left(\mathbb{N}^{n}, \leq\right)$. So, we can use interchangeably both definitions of multicomplex.

A multicomplex whose maximal elements are all of the same rank, where the rank of an element is the sum of the value of its entries, is called pure. The vector $\left(h_{0}, \ldots, h_{d}\right)$, where $h_{i}$ is the number of monomials of rank $i$, is the degree sequence of the multicomplex. A vector $\left(h_{0}, \ldots, h_{d}\right)$ is called a (pure) $O$-sequence if it is the degree sequence of some (pure) multicomplex. R. Stanley proved that the $h$-vector of a shellable simplicial complex is an $O$-sequence and made the following conjecture [Sta96].

Conjecture 4.1. The h-vector of a matroid complex is a pure O-sequence.

Now we are ready to prove the main result of this section.

Theorem 4.2. If $M$ is a cographic matroid, then the h-vector of the matroid complex $\Delta(M)$ is a pure $O$-sequence. In other words the conjecture of Stanley is true for cographic matroids.

Proof. Let $M$ be a cographic matroid. Then its dual $M^{*}$ is a graphic matroid and there exists a graph $G$ such that $M^{*}=M(G)$. Let $|V(G)|-1=n$ and $q \in V(G)$. We are going to prove that the set of critical configurations of $G, C$, can be transformed to be a pure multicomplex whose $O$-sequence is the $h$-vector of $\Delta(M)$.

First consider any critical configuration $c$ and suppose that $c^{\prime}$ is a stable configuration such that $c^{\prime} \geq$ $c$, here we consider configurations as elements of $\left(\mathbb{N}^{n}, \leq\right)$. We will prove that $c^{\prime}$ is also critical. Let $d(v)=c^{\prime}(v)-c(v)$, for $v \neq q$, and $\sigma$ any critical sequence for $c$. At each vertex $v$, we mark $d(v)$ chips as "permanent" and the rest as "movable". Then, starting from $c$ ', we can follow the legal firing $\sigma$ without problem, if we just use the "movable" chips. At the end, as $c$ is critical, we have again $c(v)$ "movable" chips in each $v \neq q$, and also $d(v)$ "permanent" chips that did not move. So, $c$ ' is a critical configuration.

From the above argument, we get that $\bigvee_{c \in \mathcal{C}} c$, the coordinate-wise maximum of all elements of $C$ in $\left(\mathbb{N}^{n}, \leq\right)$ is a critical configuration, namely the configuration defined by

$$
c_{\perp}(v)=\operatorname{deg}(v)-1, \quad v \neq q .
$$

Now, let $I$ be the down-set in $\left(\mathbb{N}^{n}, \leq\right)$ generated by $c_{\perp}$, that is, $a \in I$ if $a \leq c_{\perp}$. Clearly $C$ is contained in $I$. Consider the function $f: I \rightarrow \mathbb{N}^{n}$ given by

$$
f(c)(v)=c_{\perp}(v)-c(v) .
$$


Note that $f(c) \in I$ and $f(f(c))=c$. Also it is clear that if $c$ and $c^{\prime}$ are critical configurations such that $c \leq c^{\prime}$, then $f(c) \geq f\left(c^{\prime}\right)$.

Let $a \in f(C)$ and $b \in \mathbb{N}^{n}$ such that $b \leq a$, then $\overrightarrow{0} \leq b \leq a$ and the images of these elements satisfy $f(a) \geq f(b) \geq f(\overrightarrow{0})=c_{\perp}$. By the second inequality we conclude that $f(b)$ is a stable configuration. We know that there is a critical configuration $c$ such that $f(a)=c$, so by the preceding argument and the first inequality we get that $f(b)$ is a critical configuration and $b=f(f(b)) \in f(C)$. Therefore $f(C)$ is a multicomplex.

The rank of an element of $\left(\mathbb{N}^{n}, \leq\right)$ is just $\sum_{i=1}^{n} a(i)$. For a critical configuration $c$ of level i, $f(c)$ has rank

$$
\begin{aligned}
\sum_{v \neq q} f(c)(v) & =\sum_{v \neq q}\left(c_{\perp}(v)-c(v)\right) \\
& =\sum_{v \neq q}(\operatorname{deg}(v)-1)-\sum_{v \neq q} c(v) \\
& =2|E(G)|-\operatorname{deg}(q)-|V(G)|+1-w(c) \\
& =2|E(G)|-\operatorname{deg}(q)-|V(G)|+1-\operatorname{level}(c)-|E(G)|+\operatorname{deg}(q) \\
& =|E(G)|-|V(G)|+1-i .
\end{aligned}
$$

So, critical configurations of the same level are mapped by $f$ to elements of the same rank.

Also, observe that if $c$ is a critical configuration then there exists a critical configuration $c^{*}$ of minimal level such that $c^{*} \leq c$. First consider the case when $G$ has no loops. Take a legal sequence for $c$ that makes it recur. We know by Lemma 3.2 that this is a permutation of the vertices. We repeat this firing but this time we will label some chips. All the chips sent by $q$ are labelled $q$. When we fire vertex $v_{i}$, we mark any unmarked chip that is moved with the label $v_{i}$. Any already marked chip will return to the vertex of its label. This is possible because we have a critical configuration. Clearly, at the end, the number of marked chips that are not labelled $q$ is $|E|-\operatorname{deg}(q)$. So these marked chips induce a critical configuration $c^{*}$ of minimal level (level 0 in this case) such that $c^{*} \leq c$. In the case that $G$ has loops, , we can fix two chips for every loop and continue as in the loopless case but the level of $c^{*}$ will go up by 1 for each loop.

We conclude that all the coordinate-wise minimal elements of $\mathcal{C}$ ( or minimal critical configurations) have all the same level, and it is equal to the minimal level.

Clearly, the maximal elements of $f(C)$ are the image under $f$ of the minimal critical configurations, and as all these have the same rank, we obtain that $f(C)$ is a pure multicomplex whose pure $O$-sequence is the vector $\left(c_{|E(G)|-|V(G)|+1}, \ldots, c_{k}\right)$, where $k$ is the number of loops of $G$.

To finish the proof, we use Theorem 3.4 to get

$$
\begin{aligned}
\sum_{i=0}^{r} c_{i} y^{i} & =T(G ; 1, y) \\
& =\sum_{i=0}^{r} h_{i} y^{r-i},
\end{aligned}
$$

where $r=|E(G)|-|V(G)|+1$ is the rank of $M$. Then $c_{r-i}=h_{i}$, for $0 \leq i \leq r$, as required.

Let $G$ be a graph with $n$ vertices and let $q$ be the special vertex. If $\mathcal{C}_{q}=C$ is its set of critical configurations, then, using the notation of Theorem 4.2, we define the function $f_{\mathcal{C}}: \mathcal{C} \rightarrow \mathbb{N}^{n}$ given by

$$
f_{\mathcal{C}}(c)(v)=c_{\perp}(v)-c(v) .
$$


and $f_{C}(C)$ is a pure multicomplex that we denote by $\mathcal{M}_{q}(G)$. Note that changing the choice of $q$ may change the multicomplex but not the degree sequence.

Recently, Manoj Chari proves Theorem 4.2 but using a different technique, see [Cha00].

\section{A conjecture of Manoj Chari}

As we mentioned, using algebraic methods, Stanley [Sta96] showed that the $h$-vector of any shellable simplicial complex is an $O$-sequence. An explicit numerical characterisation for $O$-sequences is well known due to Macaulay [Sta96], and this gives a set of numerical restrictions on the set of $h$-vectors of shellable complexes.

A complete numerical characterisation is not known for pure $O$-sequences. However, Hibi has shown, see [Hib92], that a pure $O$-sequence $\left(h_{0}, h_{1}, \ldots, h_{d}\right)$ must satisfy the following conditions:

$$
\begin{gathered}
h_{0} \leq h_{1} \leq \cdots \leq h_{[d / 2]} \\
h_{i} \leq h_{d-i}, 0 \leq i \leq[d / 2] .
\end{gathered}
$$

Hibi also conjectured that the $h$-vector of a matroid complex must satisfy inequalities (5.1) and (5.2).

For the $h$-vector of a matroid complex the following result is due to Brown and Colbourn [BC92]

Theorem 5.1. The h-vector of a connected rank-d matroid satisfies the following inequalities:

$$
(-1)^{j} \sum_{i=0}^{j}(-b)^{i} h_{i} \geq 0,0 \leq j \leq d,
$$

for any positive real number $b \geq 1$ with equality possible only if $b=1$.

Note that the pure $O$-sequence $(1,4,2)$ does not satisfy $(5.3)$, so the conjecture of Stanley is a necessary condition for the $h$-vector of matroid complexes but not sufficient.

Recently, M. Chari gave a stronger result that includes the Brown and Colbourn result and solves the conjecture of Hibi, see [Cha97]. Then he made a conjecture, but to state it we require some definitions first.

Definition 5.2. A poset $Q$ is an M-poset if it is isomorphic to a multicomplex with just one maximal element.

Equivalently, an $M$-poset is a direct product of chains. Given two elements $x \leq y$ of a poset, if the interval $[x, y]$ is an $M$-poset then is called an $M$-interval.

Definition 5.3. A pure poset $P$ is $M$-partitionable if $P$ can be partitioned into $M$-intervals $\left[x_{i}, y_{i}\right], i=$ $1, \ldots, n$, such that each $y_{i}$ is a maximal element of the poset $P$. Such partition is called an M-partition of $P$ and the elements $x_{i}$ are referred to as the lower bound of the M-interval.

Observe that not every multicomplex has an $M$-partition, for example the pure multicomplex over $z_{1}, z_{2}, z_{3}$ with maximal elements $z_{1}^{2} z_{3}, z_{1} z_{2}^{2}$ and $z_{2} z_{3}^{2}$, or the pure multicomplex over $z_{1}, z_{2}, z_{3}, z_{4}$ with maximal elements $z_{1} z_{2}$ and $z_{3} z_{4}$.

Definition 5.4. An $M$-shelling of a poset $P$ is an $M$-partition of $P$, along with an ordering of the $M-$ intervals such that the union of the elements in any initial subsequence of $M$-intervals in the ordering is an order ideal of $P$. A poset $P$ is $M$-shellable if it admits an $M$-shelling. 
In the case that $P$ is a simplicial complex, $M$-shellability is equivalent to shellability; and in the case that $P$ is a (pure) multicomplex, any initial subsequence of $M$-intervals in an $M$-shelling is a (pure) multicomplex.

Being $M$-shellable is a strictly stronger notion than being $M$-partitionable as the pure multicomplex over $z_{1}, z_{2}, z_{3}, z_{4}, z_{5}$ with maximal elements $z_{1} z_{2}, z_{2} z_{3}, z_{1} z_{3}$ and $z_{4} z_{5}$ shows.

Finally, if we called shellable $O$-sequence, the degree sequence of a pure $M$-shellable multicomplex, we have Manoj Chari's conjecture:

Conjecture 5.5. For a coloop free matroid, the h-vector of its matroid complex is a shellable $O$-sequence.

Observe that the notion of $M$-shellable multicomplex is strictly stronger than that of $M$-shellable poset, see example 3 in [Cha97].

Now, we give a proof of this conjecture in the case of cographic matroids. First, we need some results about operation on multicomplexes.

For two posets $P$ and $Q$, we denote their direct product by $P \times Q$, that is, its elements are the pairs $(a, b)$ with $a \in P$ and $b \in Q$; and $(a, b) \leq\left(a^{\prime}, b^{\prime}\right)$ if and only if $a \leq a^{\prime}$ in $P$ and $b \leq b^{\prime}$ in $Q$. We have the following result from [Cha97].

Lemma 5.6. If $M_{1}$ and $M_{2}$ are $M$-shellable complexes, then $M_{1} \times M_{2}$ is an $M$-shellable complex.

If we see a multicomplex $P$ as a set of monomials over variables $z_{1}, \ldots, z_{n}$, then for a variable $x$ we denote by $x^{i} P$ the set of monomials consisting of a monomial in $P$ times $x^{i}$.

Let $P$ be a rank- $d$ multicomplex over variables $z_{1}, \ldots, z_{n+1}$ and let $Q$ be a rank- $(d-i)$ multicomplex over variables $z_{1}, \ldots, z_{n}$, with $1 \leq i \leq d$. If $z_{n+1}^{i} Q \cap P=\emptyset$ and $z_{n+1}^{i} Q \cup P$ is a multicomplex, then we called $z_{n+1}^{i} Q \cup P$ the $i$-join of $P$ with respect to $Q$ and we denoted this by $P_{i} \nearrow Q$. The definition of $i$-join exists in general for posets, see [Cha97], but we not need such generality here. Also, the following result is true for the general definition of $i$-join but here we prefer to deal just with multicomplexes.

Lemma 5.7. Let $P$ be an rank-d M-shellable multicomplex and let $Q$ be a rank-d-i M-shellable multicomplex, for a fixed $i$ with $1 \leq i \leq d$, such that $P_{i} \nearrow Q$ is defined and the maximal elements of $P$ are also maximal elements of $P_{i} \nearrow Q$. Then $P_{i} \nearrow Q$ is a rank-d M-shellable multicomplex.

Proof. Let $P_{i} \nearrow Q$ be $z^{i} Q \cup P$ for some variable $z$. Take a $M$-shelling of $P$, say $C_{1}, \ldots, C_{r}$, and a $M$-shelling of $Q$, say $D_{1}, \ldots, D_{s}$, then an $M$-shelling of $P_{i} \nearrow Q$ is $C_{1}, \ldots, C_{r}, z^{i} D_{1}, \ldots, z^{i} D_{s}$.

Now, we are ready to prove the main result of this section.

Theorem 5.8. Let $G$ be a loopless connected graph and $q$ be a special vertex of $G$, the multicomplex $\mathcal{M}_{q}(G)$ is M-shellable.

Proof. During the proof, for a graph $H$, we consider the equivalence relation over $E(G)$ given by $e \sim f$ if and only if $e$ and $f$ form a cycle of size 2; and we denote the equivalence class of $e$ by $[e]$. In other words, $[e]$ is the set of all parallel edges to $e$ together with $e$. Also, $G /[e]$ is the graph obtained form $G$ by contract all the edges in $[e]$ and $G \backslash[e]$ is the graph obtained from $G$ by removing the edges in $[e]$.

Our proof is by induction over the number of equivalence classes in $E$. The result is trivial if $G$ does not have any equivalence classes.

Let us suppose that the result is true for all graphs $H$ with at most $m$ equivalence classes. Let $G$ be a loopless connected graph with $m+1$ equivalence classes and $n+1$ vertices with $q$ the special vertex, and let $\mathcal{C}$ be the set of critical configurations of $G$ with special vertex $q$. Choose a vertex $u$ neighbour 
to $q$, so say $e=(q, u) \in E(G)$, and consider the class $[e]$. If $[e]$ is a cutset of $G$, then let $C^{\prime}$ be the set of critical configurations of $G /[e]$ with $q=u$ as a special vertex. Then for each $\theta^{\prime} \in C^{\prime}$ we obtain $|[e]|$ different critical configurations in $G$ by defining $\theta(u)=i$, for some $i \in\left[\operatorname{deg}_{G}(u)-|[e]|, \operatorname{deg}_{G}(u)-1\right]$ and $\theta(v)=\theta^{\prime}(v)$ for all $v \in V(G) \backslash\{q, u\}$. Clearly, we can fire $q$, then $u$ and then follow a critical sequence for $\theta^{\prime}$, thus $\theta$ is a critical configuration. So, the set $\mathcal{C}$ can be expressed as

$$
\mathcal{C}=\left\{\left(\theta^{\prime}, i\right) \mid \theta^{\prime} \in C^{\prime}, i \in\left[\operatorname{deg}_{G}(u)-|[e]|, \operatorname{deg}_{G}(u)-1\right]\right\},
$$

where we consider $\theta^{\prime} \in C^{\prime}$ an element of $\mathbb{N}^{(n-1)}$ ( $G /[e]$ has $n$ vertices). Thus, $\mathcal{M}_{q}(G)$ is isomorphic to the direct product of $\mathcal{M}_{q}(G /[e])$ with a chain of size $|[e]|$. The result holds for $G$ by Lemma 5.6.

Now, suppose that $[e]$ is not a cutset. We partition the set $\mathcal{C}$ of critical configurations of $G$ in two sets. One $\mathcal{A}$, the set of critical configurations $\theta$, such that $\theta(u) \in[\operatorname{deg}(u)-|[e]|, \operatorname{deg}(u)-1]=I$; and the other $\mathcal{B}$, the rest of the critical configurations.

Firstly, we partition further $\mathcal{A}$ in the sets $\mathcal{A}_{i}$ of critical configurations such that $\theta(u)=i$, for $i \in I$. Clearly, for a fixed $i \in I$, each $\mathcal{A}_{i}$ can be put into correspondence with the set $C^{\prime}$ of critical configurations of $G /[e]$ with special vertex $q=u$, by associating, to each $\theta \in \mathcal{A}_{i}$ the configuration $\theta^{\prime}$ defined by $\theta^{\prime}(v)=\theta(v)$ for all $v \in V(G) \backslash\{q, u\}$. The reader can check that $\theta^{\prime}$ is a critical configuration of $G /[e]$ and that this is a bijection. Even more, $f_{\mathcal{A}_{i}}\left(\mathcal{A}_{i}\right)$ and $\mathcal{M}_{q}(G /[e])=f_{C^{\prime}}\left(C^{\prime}\right)$ are isomorphic multicomplex ( the previous bijection followed by $f_{\mathcal{C}^{\prime}}$ is an isomorphism. Thus by the inductive hypothesis, $f_{\mathcal{A}_{i}}\left(\mathcal{A}_{i}\right)$ is an $M$-shellable multicomplex. Also, $\mathcal{A}$ can be seen as the set

$$
\left\{\left(\theta^{\prime}, i\right) \mid i \in I, \text { and } \theta^{\prime} \in C^{\prime}\right\} .
$$

So, $f_{\mathcal{A}}(\mathcal{A})$ is the direct product of $\mathcal{M}_{q}(G /[e])$ with a chain of size $|[e]|$. Therefore, $f_{\mathcal{A}}(\mathcal{A})$ is an $M$-shellable complex by Lemma 5.6.

Secondly, the critical configurations $\mathcal{B}$ can be put into correspondence with the set $\mathcal{C}^{\prime \prime}$ of critical configurations of $G \backslash[e]$ with $q$ as a special vertex. For $\theta^{\prime \prime} \in C^{\prime \prime}$, we define $\theta$ by $\theta(v)=\theta^{\prime \prime}(v)$ for all $v \in V(G) \backslash\{q\}$. It is not difficult to see that $\theta \in \mathcal{B}$ and that this is a bijection. Even more, $\mathcal{M}_{q}(G \backslash[e])$ and $f_{\mathcal{B}}(\mathcal{B})$ are isomorphic multicomplexes. Thus, by inductive hypothesis, $f_{\mathcal{B}}(\mathcal{B})$ is an $M$-shellable multicomplex.

Now, observe that $f_{\mathcal{A}}(\mathcal{A})$ is a multicomplex of rank $d=m^{\prime}-n-1$, where $m^{\prime}$ is the number of edges of $G$, and $f_{\mathcal{B}}(\mathcal{B})$ is a multicomplex of $\operatorname{rank} d-|[e]|$ (as $G$ is connected and $[e]$ is not a cut set, $|[e]| \leq d$ ). So, clearly, the multicomplex $\mathcal{M}_{q}(G)$ is the $|[e]|$-join of $f_{\mathcal{A}}(\mathcal{A})$ with respect to $f_{\mathcal{B}}(\mathcal{B})$. The proof will be complete by Lemma 5.7 if the maximal elements in $f_{\mathcal{A}}(\mathcal{A})$ are maximal elements in $\mathcal{M}_{q}(G)$. This is equivalent to prove that the minimal elements in $\mathcal{A}$ are minimal elements in $\mathcal{C}$.

Let $\theta$ be a minimal element of $\mathcal{A}$, then $\theta \in \mathcal{A}_{i}$ for $i=\operatorname{deg}(u)-|[e]|$ and by our bijection between $\mathcal{A}_{i}$ and $C^{\prime}$, the critical configurations of $G /[e]$, there exists $\theta^{\prime} \in C^{\prime}$ with $\theta^{\prime}(v)=\theta(v)$ for all $v \in V(G) \backslash\{q, u\}$. Because $\theta$ is minimal, $\theta^{\prime}$ is minimal in $C^{\prime}$, thus it has level 0 (notice that $G$ and $G /[e]$ do not have loops). Therefore,

$$
w\left(\theta^{\prime}\right)=|E(G /[e])|-\operatorname{deg}_{G /[e]}(q)
$$

and $w(\theta)=w\left(\theta^{\prime}\right)+\operatorname{deg}_{G}(u)-|[e]|$. But

$$
\begin{aligned}
\operatorname{deg}_{G /[e]}(q) & =\operatorname{deg}_{G}(q)+\operatorname{deg}_{G}(u)-2 v(q, u) \\
& =\operatorname{deg}_{G}(q)+\operatorname{deg}_{G}(u)-2|[e]|,
\end{aligned}
$$


and $|E(G /[e])|=|E(G)|-|[e]|$. We conclude that

$$
\begin{gathered}
w(\theta)=|E(G)|-|[e]|-\left(\operatorname{deg}_{G}(q)+\operatorname{deg}_{G}(u)-2|[e]|\right)+\operatorname{deg}_{G}(u)-|[e]| \\
=|E(G)|-\operatorname{deg}_{G}(q) .
\end{gathered}
$$

So, $\theta$ has level 0 in $C$ and it is minimal.

Corollary 5.9. For a coloop free cographic matroid $M$, the h-vector of its matroid complex is a shellable O-sequence.

Proof. Let $M$ be a coloop free cographic matroid and $G$ a loopless graph such that $M^{*}(G)=M$. For a vertex $q$ of $G$, Theorem 4.2 says that $\mathcal{M}_{q}(G)$ is a pure multicomplex whose degree sequence is the $h-$ vector of $\Delta(M)$, and by Theorem $5.8, \mathcal{M}_{q}(G)$ is $M$-shellable. Thus, the degree sequence of $\mathcal{M}_{q}(G)$ is a shellable $O$-sequence.

\section{References}

[ALS $\left.{ }^{+} 89\right]$ R.J. Anderson, L. Lovász, P. Shor, J. Spencer, É. Tardos, and S. Winograd. Disks, balls, and walls: analysis of a combinatorial game. Amer. Math. Monthly, 96(6):481-493, 1989.

[BC92] J. I. Brown and C. J. Colbourn. Roots of the reliability polynomial. SIAM J. of Discrete Math, 5(4):571-585, 1992.

[Big99a] N. Biggs. Chip firing and the critical group of a graph. J. Algebraic Combin., 9(1):25-45, 1999.

[Big99b] N. Biggs. The Tutte polynomial as a growth function. J. Algebraic Combin., 10(2):115-133, 1999.

[Bjö92] A. Björner. Homology and shellability of matroids and geometric lattices. In N. White, editor, Matroid Applications, Encyclopedia of Mathematics and its Applications, pages 226-283. Cambridge Univ. Press, 1992.

[BLS91] A. Björner, L. Lovász, and P. W. Shor. Chip-firing games on graphs. European J. Combin., 12:283-291, 1991.

[BO92] T. Brylawski and J. Oxley. The Tutte polynomial and its applications. In N. White, editor, Matroid Applications, pages 123-225. Cambridge Univ. Press, 1992.

[BW97] N. Biggs and P. Winkler. Chip-firing and the chromatic polynomial. Research Report LSECDAM-97-03, CDAM, 1997.

[Cha97] M. K. Chari. Two descompositions in topological combinatorics with applications to matroid complexes. Trans. Amer. Math Soc., 349(10):3925-3943, 1997.

[Cha00] M. K. Chari. Acyclic orientations and the reliability polynomial. Draft, October 2000.

[CR00] R. Cori and D. Rossin. On the sandpile group of dual graphs. European J. Combin., 21(4):447459, 2000. 
[Hib92] T. Hibi. Face number inequalities for matroid complexes and Cohen-Macaulay types of Stanley-Reisner rings of distributive lattices. Pacific Journal of Math., 154:253-264, 1992.

[Mer97] C. Merino. Chip-firing and the Tutte polynomial. Ann. Comb., 1(3):253-259, 1997.

[Ox192] J.G. Oxley. Matroid Theory. Oxford Univ. Press, 1992.

[Spe86] J. Spencer. Balancing vectors in the max norm. Combinatorica, 6:55-66, 1986.

[Sta96] R.P. Stanley. Combinatorics and Commutative Algebra, volume 41 of Progress in mathematics. Birkhuser, Boston-Besel-Stuttgart, 2nd edition, 1996.

[Wel76] D.J.A. Welsh. Matroid Theory. Academic Press, 1976. 
